\title{
Research on path planning of mobile robot based on improved ant colony algorithm
}

\author{
Wang Rui ${ }^{1, a}$, Wang Jinguo ${ }^{2, b^{*}}$ (corresponding author), Wang $\mathrm{Na}^{3, \mathrm{c}}$ \\ ${ }^{1}$ Department of Information Engineering, Jilin Business and Technology College \\ China \\ ${ }^{2}$ Department of Urology, the First Hospital of Jilin University, China \\ ${ }^{3}$ Department of Anaesthesiology, the First Hospital of Jilin University, China \\ aXiaoben6666@126.com, ${ }^{b}$ wangjinguolily@163.com, ${ }^{c}$ lilyly12345@163.com
}

\section{Key words: Mobile robot, Path planning, Ant colony algorithm}

\begin{abstract}
At present, the problem of global path planning research most concentrated in the known information conditions. But the path planning of mobile robot is still an important problem to be solved in dynamic environment. In this paper, the ant colony algorithm is improved to solve the robot path planning problem in the unknown environment.
\end{abstract}

\section{Introduction}

In the early 90 century, Italy scholar M.Dorigo [1] proposed the ant colony algorithm, inspired by the foraging behavior of ants in nature. As the ant colony algorithm proposed, many scholars have a great interest in it. The application of the ant colony algorithm is the combination of optimization problem initially. Currently the application of ant colony algorithm has gradually extended to engineering science and technology field, such as the traveling salesman problem(TSP)、 scheduling problem, graph coloring problem, communication network routing problem, vehicle routing problem, mobile robot path planning and so on[2].

Ant colony algorithm is a typical local path planning algorithm. In the process of searching the path, each ant is based on the information of its environment, and carries out real-time route planning according to some simple rules[3]. Although each ant acts a simple behavior, many ants in the ant colony that interact with the environment will show a complex and flexible behavior.

The advantages of the ant colony algorithm: It has a strong robustness: the ant colony algorithm model can be modified to resolve other problem[4]. It is an essentially parallel algorithm. Because of large-scale parallel computing, it can significantly reduce the computation time. It is a positive feedback algorithm. The positive feedback accelerates the search convergence. Ant algorithm is easy to combine with a variety of heuristic algorithms to improve the performance of the algorithm[5].

However, this algorithm also has some defects,Compared with other methods, this algorithm generally takes longer search time[6]. Although the speed of computer 
computing and the nature of ant colony algorithm can reduce some problem. But this is still a big obstacle for some large-scale optimization problems. In addition, the ant colony algorithm is easy to appear stagnation behavior. After searching to some extent, all the individuals have the same solution. It cannot be further searching for the rest solution space, so it cannot find a better solution. In this paper, we will improve the ant colony algorithm, and the improved algorithm will overcome its defects, and make it have faster convergence.

\section{Improvement of algorithm}

In order to guarantee the algorithm can converge to the global optimal solution or approximate optimal solution and solve the deadlock problem, the following improvement is done in this chapter.

Transfer probability adjustment. In order to increase the multiplicity of solutions, we can use the random selection strategy in the transfer probability. First, we set a random selection parameter $q_{0} \in(0,1), \mathrm{q}$ is a random number between 0 and 1 . When $q<q_{0}$, we select any feasible node around the current node $\mathrm{r}$ randomly. Otherwise, the select the next feasible node according formula(1).

$n=\left\{\begin{array}{lr}\operatorname{rand}_{\left(\text {allowed }_{k}\right)} \quad q<q_{0} \\ n & \text { otherwise }\end{array}\right.$

In which, rand(allowedk) represents to selects a node randomly from allowedk, $\mathrm{n}$ is represented the next node chosen by the transfer probability formula. In addition, two heuristic factors are added to the transfer probability formula in order to enhancing the global search capability of the improved ant colony algorithm. The new transfer probability formula is shown below:

$$
p_{r n}^{k} \begin{cases}\frac{\left[\tau_{r n}(t)\right]^{\alpha}\left[\eta_{r n}(t)\right]^{\beta} S_{n}^{-1} G_{n}^{-1}}{\sum_{n \in \text { allowed }_{k}}\left[\tau_{r n}(t)\right]^{\alpha}\left[\eta_{r n}(t)\right]^{\beta} S_{n}^{-1} G_{n}^{-1}} & s \in \text { allowed }_{k} \\ 0 & \text { otherwise }\end{cases}
$$

In which, $\mathrm{Sn}$ is a distance that from the nodes $\mathrm{n}$ to the target point. Gn represents the sum of times that the next visited $\mathrm{n}$ node. After the ants visit $\mathrm{n}$ node, the Gn adds 1. From the formula (2) can see, the $p_{r n}^{k}$ is inversely proportional to $\mathrm{Sn}$ that the next node are closer to the target node, the node has the higher probability to be chosen. It can accelerate the convergence rate. The $p_{r n}^{k}$ is inversely proportional to $\mathrm{Sn}$ that the more times number of the next nodes to be selected, the smaller the probability of the node is selected. Its purpose is to find a new path, increase the diversity of solutions, so that the probability of falling into local optimum is reduced. 
Update pheromone. In order to further reduce the probability of the local optimal solution to the ant colony algorithm, improve the global search ability of the algorithm, we borrow MMAS features. After the end of each generation cycle, The upper and lower bound of the pheromone is introduced when the intensity of the pheromone is updated. The limit principle of pheromone is as follows:

$$
\Delta \tau_{i j}^{k}=\left\{\begin{array}{l}
Q\left(1-r_{k} / R_{k}\right) \quad \text { antk transfer from } i \text { to } j \\
0 \quad \text { otherwise }
\end{array}\right.
$$

So it will get the local pheromone updating rule as follow:

$$
\begin{aligned}
& \tau_{i j}^{\prime \prime}=\rho * \tau_{i j}^{\prime}+\Delta \tau_{i j} \quad \forall i, j \quad i \neq j \\
& \Delta \tau_{i j}=\sum_{k=1}^{m} \Delta \tau_{i j}^{k} \\
& \tau_{i j}^{\prime} \text { and } \tau_{i j}^{\prime \prime} \text { represent the original pheromone concentration and update pheromone }
\end{aligned}
$$

concentration. With introducing the local pheromone updating, it can limit the pheromone accumulation of the path. By adjusting the $\mathrm{Q}$ value,we can adjust the radio of search same path by other ants. It will expand the scope of the search and reduce the radio of search falling into the local optimal solution.

Deadlock handling. In some complex environments, such as it has many obstacles and most of them present the "U" or "V" type, mobile robot is difficult to avoid these obstacles so it is in deadlock state. This paper presents a "fallback - punishment" strategies to solve the problem of deadlock. The node in deadlock position is deadlock node. The edge in deadlock node is deadlock edge. The idea of "Fallback-punishment" strategy is when one ant in a deadlock state, the ant not to death, but allows regression step. We need to update the ant's tabu list, remove the deadlock node from the tabu list, then, punish the pheromone on the edge of the deadlock, that is, to reduce the pheromone on the deadlock path. It will avoid other ants to fall into a deadlock state and reduce the search time. Finally, ants choose the new node on the current path. The pheromone punish can be considered as a local pheromone update, and the pheromone penalty function is shown below:

$\tau_{i j}=(1-\lambda) \tau_{i j}$

In which, $1-\lambda$ is a pheromone punishment coefficient. If ants were trapped in deadlock state in the early days, then other ants would be few trapped in deadlock again in the same position. So the deadlock problem is solved and improve the global search ability of ant colony algorithm.

\section{Algorithm simulation and results analysis}

The simulation uses the $200 * 200$ grid to divide the environment space. The robot sensor radius is $r=10$, the step size is 4 . We adopt the algorithm parameters as follow: $\alpha=1, \beta=3, \rho=0.8$. The ants number is $\mathrm{m}, \mathrm{m}=100$. The maximum iteration 
number is $\mathrm{nc}, \mathrm{nc}=50$.We use the improved ant colony optimization for the mobile robot path planning,the results as shown in figure 1.

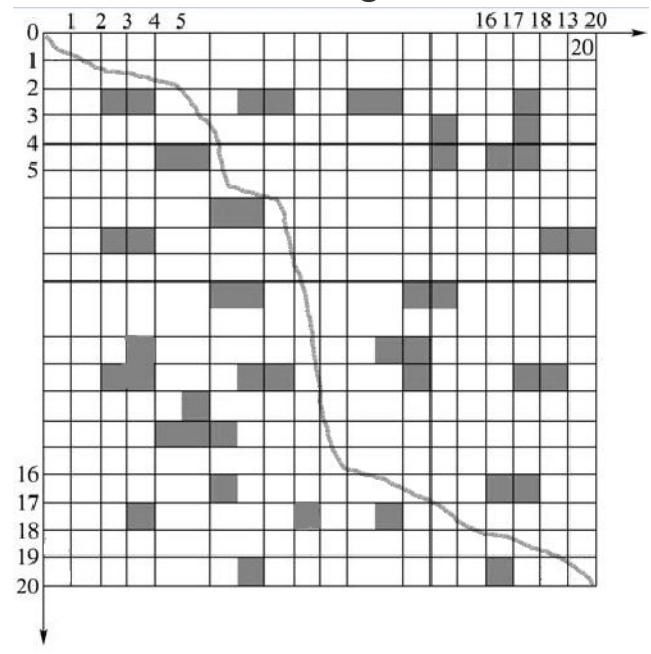

Fig1.the path planning of the improved ACO

\section{Conclusion}

In this paper, we propose an improved ACO algorithm. In order to increasing the algorithm' s convergence speed and avoiding to fall into local optimum, we propose adaptive migratory probability function and updating the pheromone. We apply the improved algorithm to path planning for mobile robot and the simulation experiment proved that improved algorithm is viable and efficient.

\section{References}

[1] ZHANG Xue-min, ZHANG Hang. Research An Improved Ant Colony Algorithm of the Optimal Routing Problem[J]. Techniques of Automation \& Applications(2009)

[2] DENG Wang-yu,ZHENG Qing-hua,CHEN Lin,et al.Research on extreme learning of neural networks[J].Chinese Journal of Computers,2010,33(2):279-287.

[3] BI Xiao-jun,LUO Guang-xin. The improvement of ant colony algorithm based on the inver-over operator[A].IEEE International Conference on Mechatronics and Automation [C].Harbin:IEEE Press,2007.

[4] Garcia M A Porta,Montiel Oscar,Castillo Oscar,et al.Path planning for autonomous mobile robot navigation with ant colony optimization and fuzzy cost function evaluation[J].Applied Soft Computing,2009,9(3):1102-1110.

[5] Min Gyu Park,Jae Hyun Jeon,Min Cheol Lee.Obstacle avoidance for mobile robots using artificial potential field approach with simulated annealing. IEEE International Symposium on Industrial Electronics . 2001

[6]Zheng LIU,Marcelo HANG Jr,Winston Khoon Guan SEAH.A Potential Field Based Approach for Multi-Robot Tracking of Multiple Moving Targets. The First Humanoid, Nanotechnology, Information Technology, Communication and Control, Environment and Management (HNICEM)International Conference . 2003 